\title{
Integration of the analysis of the
} error of geometric dimensions modeled with a probabilistic approach

\author{
Marc GILLE \\ PHIMECA Engineering \\ Cournon d'Auvergne, France \\ SIGMA Clermont-Ferrand \\ Clermont-Ferrand, France \\ gille@phimeca.com \\ Pierre BEAUREPAIRE \\ SIGMA Clermont-Ferrand \\ Clermont-Ferrand, France \\ pierre.beaurepaire@sigma-clermont.fr
}

\author{
Fabien TAGHON \\ PHIMECA Engineering \\ Cournon d'Auvergne, France \\ taghon@phimeca.com \\ Antoine DUMAS \\ PHIMECA Engineering \\ Cournon d'Auvergne, France \\ dumas@phimeca.com
}

\author{
Nicolas GAYTON \\ SIGMA Clermont-Ferrand \\ Clermont-Ferrand, France \\ nicolas.gayton@sigma-clermont.fr \\ Thierry YALAMAS \\ PHIMECA Engineering \\ Cournon d'Auvergne, France \\ yalamas@phimeca.com
}

\begin{abstract}
In this paper, we present two methods that complement each other, which can be used by metrologists to consider the measurement errors. The first one deals with distributions of set of measures to provide a new reference distribution. The second one uses Bayesian statistics: for each measure of a batch, it returns the most probable true dimension associated with. The outcomes of these procedures describe more faithfully the true dimensions, as the impact of the measurement errors is partially reduced. Lastly, analysis tools are proposed to evaluate the actual production risks and to be able adjust them consciously.

Synthese - Deux méthodes sont présentées dans ce papier pour que les métrologistes puissent prendre en compte l'erreur de mesure comme une variable aléatoire. La première traite la loi de probabilité qui caractérise un échantillon de plusieurs mesures et en renvoie une nouvelle, de référence. Ce résultat est utilisé en entrée de la seconde méthode qui utilise les statistiques bayésiennes pour prédire pour chaque mesure la valeur vraie la plus probable. Ces méthodes réduisent l'impact des erreurs de mesures pour mieux prédire les vraies valeurs mesurées. Enfin, des outils d'analyses sont proposés afin d'évaluer et d'ajuster consciemment les risques de production.
\end{abstract}

\section{Keywords-Metrology, Uncertainties, Statistics, Bayesian}

\section{INTRODUCTION}

Metrology is a key stage in industry as it validates the quality requirements at different steps of the production process. Designers prepare a Computer-Aided Design (CAD) model of the parts; a nominal value and tolerance interval are associated with each dimension (such as for instance the thickness of the part, the diameter of a hole, the radius of a fillet, etc.). Off-tolerance dimensions may have detrimental effects, for instance two mating parts need to be geometrically compatible to perform assembly. These dimensions are measured when the parts are manufactured. However, as every measure is always tarnished with errors, the issue of the relevance of such measures arises.

The consequence of an improper measure gives a wrong idea of the part conformity. It may lead to additional machining, scraping of compliant parts, or delivery of noncompliant parts to a customer. When studying a batch of parts, all of these dimensions are used to compute some quality metrics - such as the well-known $C_{p}$ and $C_{p k}$, thus also subject to errors. Therefore, the errors also propagate to these quality metrics.
To the authors' knowledge, the measure process currently takes into account the measurement tool accuracy. A common practice consists in applying the maximum accuracy error on both side of the measure to create the tolerance interval. However there is actually no reason to center the error interval on the measure. Moreover, only the bounds of the error interval are retained in the end, suggesting that all errors inside this interval are equiprobable. This actual lack of knowledge can be seen as a uniform probability density function modeling of the error.

If the measurement tool accuracy is mistaken, many other error sources are currently missing. This can be studied with the Ishikawa diagram which analyzes the method, environment, material, process and people sources of uncertainties [1]. The temperature, pressure, dust, altitude, operator repeatability and inter-operability are all example of errors that are being ignored. If metrologists are actually aware of these input errors, there is nowadays no mean to integrate them. By default, a certain amount of skepticism is introduced into the safety factors to prevent any risks, and the cost of these coefficients has already been widely stated in the literature.

This paper aims to use all the available information. The error is modeled as a random variable and its probability 
density function is considered as a given input. This research is part of the European project STAM [Acknowledgments] leading by an expert metrology company where engineers assume today to be able to identify it. The measure is the value that can be read on the measurement tool. This work aims at correcting (at least partially) the effects of the error on the measure, and tries to estimate the true dimension. The search for the true dimension is based on this fundamental formula [1]:

$$
m=v+\varepsilon
$$

where $m \in \mathrm{R}$ is the measure of the true dimension $v$ tarnished with the error $\varepsilon$. The true dimension is a notion that needs to be carefully manipulated since it is actually impossible to know its exact value; many metrologists believe it is very complex to define it. The uncertainties around the manufacturing process cannot be avoided, therefore, the made parts have not exactly the same dimension. That is why the true dimension is considered as a random variable.

The paper finally aims to solve two main issues: (i) the correction of the density associated with a batch of measures; (ii) the correction of the measure associated with one part. Two methods that complement each other are developed and illustrated with an example. This study assumes that the error and measure input probability density functions are Gaussian as it is widely used and covers most of the practical cases. Note that this is particularly relevant for the error as it is the sum of numerous independent variables. The use of Gaussian distribution is therefore in good agreement with the central limit theorem. This paper develops the methodology in Section II, Section III presents an example of their application and the paper closes with the conclusions.

\section{PROPOSED METHODS OF ANALYSIS}

\section{A. Correction of the density associated with a batch of measures}

A manufactured part is meant to verify some key dimension expectations. To check the quality of a batch of parts, these essential dimensions are measured. The distributions obtained are all the more important that they are used to establish the quality metrics, such as process capability indexes [2][3]. However, just as a measure is always tarnished with an error, the distribution of a set of measures is as well tarnished with errors. To estimate the true dimension distribution, the idea is to exploit the error distribution we assumed to know. The measure dimension is expressed as the sum of two random variables and its probability density function is expressed as:

$$
f_{M}(z)=\int_{-\infty}^{+\infty} f_{V}(x-z) f_{\varepsilon}(z) d z
$$

where $f_{M}$, is the probability density function of the measure, $f_{V}$ and $f_{\varepsilon}$ are respectively the probability density functions of the two input random variables: the true dimension $V$ and the error $\varepsilon$. This formula corresponds to the measure distribution and it happens to be a convolution product. Convolution is a well-documented issue, notably resolvable with a Monte Carlo simulation or Gauss' integration scheme [4]. In our context, the convolution product is known - the results are available (measures) - and one term of the product is also known the probability density function of the error. The identification of the second term of the convolution product is referred to as deconvolution.
Deconvolution methods to deal with continuous signals, either temporal or spatial, are mainly used and developed in image processing [5] and signals processing [6][7]. The metrological approach is not about filtering: a sample set is available, it is not time-dependent and each measure is a unique phenomenon. Therefore, these well documented methods are not applicable to the deconvolution of probabilistic distributions.

We recall that we introduced the assumption of a Gaussian modeling. In this context the most relevant method in our context is the use of characteristic functions, as they provide an analytical solution. If a random variable has a probability density function, then the characteristic function is its inverse Fourier transform. As a Gaussian distribution satisfies the conditions of Bochner's theorem [9], its characteristic function exists and its expression is [8]:

$$
\Phi_{X}(z)=e^{i \mu_{X} z-\frac{1}{2} \sigma_{X}^{2} z^{2}}
$$

with $\mu_{X}$ and $\sigma_{X}$ respectively the mean and the standard deviation of the normal distribution which characterize the random variable $X$. Then is used the interesting property that the characteristic function of the sum of two independent random variables is equal to the product of each of their characteristic function [11]. As we can assume the independence between the true dimension $\mathrm{V}$ and the error $\varepsilon$, the relation obtained is:

$$
\Phi_{M}(z)=\Phi_{V+\varepsilon}(z)=\Phi_{V}(z) \Phi_{\varepsilon}(z)
$$

This last expression makes the deconvolution straightforward: the characteristic function of the true dimension density is obtained by a division. The division of the two exponential functions gives an exponential function with the same shape, from which it is very easy to identify the parameters. The true dimension distribution that we finally get is Gaussian; its mean is the measure mean minus the error mean; its variance is the measure variance minus the error variance.

$$
V \sim \mathcal{N}\left(\mu_{M}-\mu_{\varepsilon}, \quad \sigma_{M}^{2}-{\sigma_{\varepsilon}}^{2}\right)
$$

\section{B. Correction of the measure associated with one part}

The first method deals with the distribution of the measure, as we considered a batch of parts. This subsection describes another method which is focused on the measure of each part of the batch. The main objective is to determine if the parts respect the tolerance specifications, and more generally for each part the probability that the dimension lays within its tolerance interval. It is impossible to know the true dimension density, so this method aims to get an approximation of it, at least better than the measure which is always tarnished with errors.

Recall that the measure has been introduced as the sum of a true dimension and an error. So, each measure can be associated with an infinite number of combinations of true dimension and error. However, the combinations are not all equiprobable. The research consists in finding the most probable couple of terms of which the sum is equal to the measure. On one side, the error measurement probability function is obviously used to weigh the plausibility of a supposed error. On the other side, it is necessary to provide 
some other new information for the a priori to estimate the plausibility of a supposed true dimension.

The production team possesses a specific expertise, which includes some theories and a long-time experience for operators and engineers, as long as the machine in the manufacturing process is maintained and keeps working to make the investment the most profitable. This non negligible knowledge exists and just waits to be picked up. Nevertheless, it must be noticed that it remains very complex to quantify the truthfulness and integrity of this source. Another interesting alternative is to use the deconvoluted distribution from the last application as the a priori probability density function. Like the production team's knowledge, this shall identify the production behavior, with an experience based on a previous huge batch of parts and being "conscious" of the error probability density function. This is the a priori information chosen in this paper. Remark that the batch being corrected must be independent from the one used for the deconvolution [10]. In order to assimilate this knowledge, we use the Bayesian statistics. From these knowledge sources, the Bayesian theorem [10] highlights the probability for a given value $\mathrm{x}$ to be the true dimension knowing the measure $\mathrm{m}$ is given by:

$$
P(x \mid m)=\frac{1}{C} \mathcal{L}(m-x) \pi(x)
$$

where $\mathscr{L}$ is the likelihood, $P$ and $\pi$ are respectively the a posteriori (or posterior) and the a priori (or prior) probability density functions, and $C$ is the evidence which guarantees that the a posteriori distribution integrates to one. The a priori distribution corresponds to the expert knowledge which weighs the plausibility of a supposed true dimension. It would be the distribution that characterizes the manufacturing process if no measure were made. The likelihood gives the probability for $m$ to have been measured knowing that the true dimension is $x$. It is based on the error density, which is known, we get: $\quad \mathcal{L}(m-x)=\varepsilon(x-m)$. The likelihood would be used to compute the probability of being out-of-tolerance if there was no a priori.

The outcome of the procedure for each measure is an a posteriori distribution, which contains much more information. In particular, the highest density of the a posteriori is called the revised dimension: this is the most probable true dimension. While the traditional use of the Bayesian theorem is meant to update the a priori with the observations, we may say that we update our observations with our a priori. This Bayesian approach can easily be extended to any arbitrary probability density function. In our context, the input distributions are supposed to be Gaussian. So, the product between the likelihood and the a priori is a density product of two Gaussian distributions which can be solved analytically. The posterior distribution obtained is also Gaussian and, incorporating the previous expression of the likelihood and that the a priori input is set as a deconvoluted distribution, it is expressed as:

$$
R_{m} \sim \mathcal{N}\left(m\left(1-\frac{\sigma_{\varepsilon}^{2}}{\sigma_{M}{ }^{2}}\right)+\frac{\sigma_{\varepsilon}^{2}}{\sigma_{M}^{2}} \mu_{M}-\mu_{\varepsilon}, \quad \sigma_{\varepsilon}{ }^{2}\left(1-\frac{\sigma_{\varepsilon}{ }^{2}}{\sigma_{M}{ }^{2}}\right)\right)
$$

where $R_{m}$ is the random variable of the revised dimension. It is worth noting that there is a linear relationship between the mean of the revised distribution and the measure. Also, the standard deviation is constant and does not depend on the input measure.

\section{EXAMPLES}

\section{A. Presentation of the example}

To illustrate the effect of these two methods we consider the following analytical examples; the data (measure) are generated using a Monte Carlo simulation, which allows us to compare the results to the known solution, thus, to quantify their quality.

The first major input is the error probability density function. Recall that it is assumed to be known using the metrologist expertise. It is important to remind that this information is actually impossible to identify perfectly. Yet to estimate the extent of the method potential, the error probability density function is supposed to describe perfectly the mistakes made in reality. This probability density function used here is unbiased, in the example its mean is equal to zero. Bias correction is straight-forward and already applied in industry, this is therefore not considered here.

The second major input is the true dimension probability function which is also set arbitrarily. Its mean value is not discussed in detail as it does not influence the study. The standard deviation is set in order to be twice the standard deviation of the error. If the measurement tools have the same dispersion than the production - which is very unlikely - then they are useless. On the contrary, if they are too precise then there may be no need for the application of these methods.

This sample size is set to 1000 . It is moderately large from a statistical perspective and we assume that the uncertainties are fairly well represented. On the other hand, collecting and measuring 1000 dimensions requires quite some efforts for a real-life manufactured component. We therefore assume that our sample size is a fair trade-off between the statistical relevance and industrial constraints.

To get a measure sample, two samples are drawn from the error and the true dimension probability density function and added together as a whole. The limits of the tolerance interval are set to 14.0 and 14.4. Recall that the input distributions are assumed to be Gaussian. Table 1 summarizes the input distributions:

TABLE I. PARAMETERS OF THE INPUT DISTRIBUTIONS

\begin{tabular}{|l|c|c|}
\hline \multicolumn{1}{|c|}{ Probability density function } & Mean & $\begin{array}{c}\text { Standard } \\
\text { deviation }\end{array}$ \\
\hline True dimension & 14.2 & 0.1 \\
\hline Error & 0.0 & 0.05 \\
\hline
\end{tabular}

\section{B. Deconvolution of a batch of parts}

The methods described in Section II.A are applied to remove the effects of the errors to the distribution identified from a set of measures. The Figure 1 shows the deconvolution result. The deconvoluted line is very close to the solution - the true dimension density. Despite an analytical solving of the problem, the deconvolution probability density function does not exactly match the solution. This difference is only due to the sample size, as the mean and variance of the measure sample differ slightly from those of the law from which it is extracted. Both the mean and variance of the deconvoluted distribution are still very satisfying: the error made for these 
parameters is respectively about 0.059 and 0.001 times the standard deviation. Nevertheless, the high quality of the results obtained must be put in parallel with the Gaussian modeling hypothesis and the perfect identification of the error probability density function that have been assumed.
The deconvoluted distribution can be subsequently used as the reference to characterize the production process. The reestimation of the parameters directly impacts the percentage for parts to be out-of-tolerance. This is explicitly shown in the Figure 2 where the probability goes from 0.0736 with the measures to 0.0462 with the deconvolution.

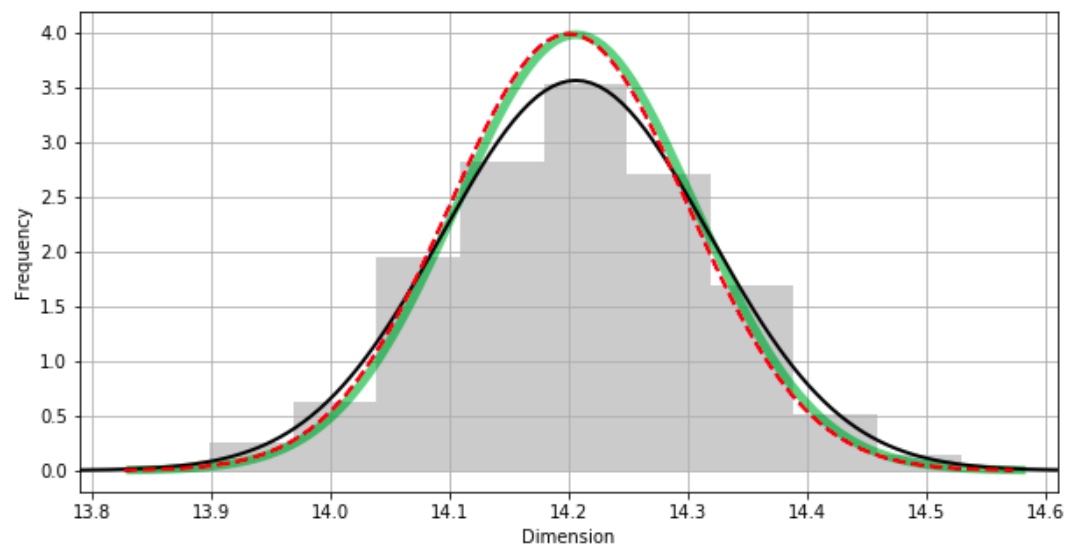

Figure 1: Deconvolution of the density associated with a batch of parts.

The dashed red line is the true dimension distribution. The black line is the Gaussian distribution inferred on the measure sample represented by the transparent grey histogram. The thick green line is the deconvoluted distribution.

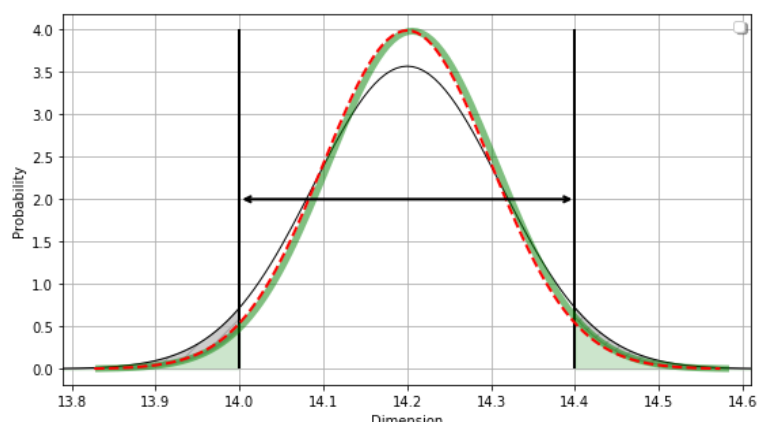

(a)

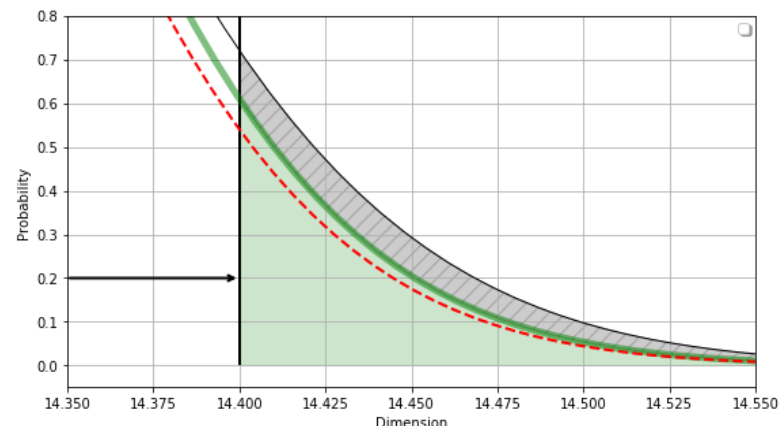

(b)

Figure 2: Probability for the process to produce a part out-of-tolerance.

Graph (b)focuses on the right side of the graph (a), to explicit the probability failure reduction. The red dashed line is the true dimension density, the large green line is the deconvoluted density and the solid black line is the measured dimension density. The black arrow indicates the tolerance interval bounds, the green area and the grey striped area are the probability to be out of it, respectively according to the deconvoluted distribution and to the measured distribution.

\section{Revision of the measure associated with a part}

The methods described in Section II.B are applied to remove the effects of the errors to each measure of a simulated set. The deconvoluted distribution from the last application is set as the a priori probability density function. The scatter plot of the measure residues in Figure 3.a shows a concentric dispersion which confirms the fair construction of the sample, based on two Gaussian probability density functions. The scatter of the revised dimension shown in Figure 3.b presents a dispersion smaller than the measured ones: the mean squared error goes from 1.555 to 1.431 . It also presents a linear decreasing tendency, pointing out the dependence between the revised dimension and the true dimension value. This is clearly visible for the extreme values of the true dimension. The introduction of a bias is the price of an improvement of the measure.
The coefficient of determination is used to quantify the improvement introduced by the proposed method. This metrics is frequently used in the context of (non-)linear regression, as it can be considered as the score of the predictive model. The idea in this application is to consider that the measure and revised dimension can be seen as predictions of the true dimension:

$$
R^{2}=\frac{\sum_{i=1}^{n}\left(x^{(i)}-\mu_{V}^{2}\right)^{2}}{\sum_{i=1}^{n}\left(v^{(i)}-\mu_{V}^{2}\right)^{2}}
$$

where $x$ is either the measure or the revised dimension, $\mu_{V}$ is the mean of the true value dimension distribution. If one of these two predictions is perfect then its predicted value is equal to the true dimension and the score is equal to 1 . The uncertainties present in this model make the search of almost perfect values irrelevant. The coefficient of determination computed in this example on the measured and revised 
dimensions are respectively equal to 0.776 and 0.811 . This result is satisfying as it endorses the general increase in quality.

The mean squared errors and the coefficients of determination confirm that the revised dimensions obtained through Bayesian statistics are generally better than the

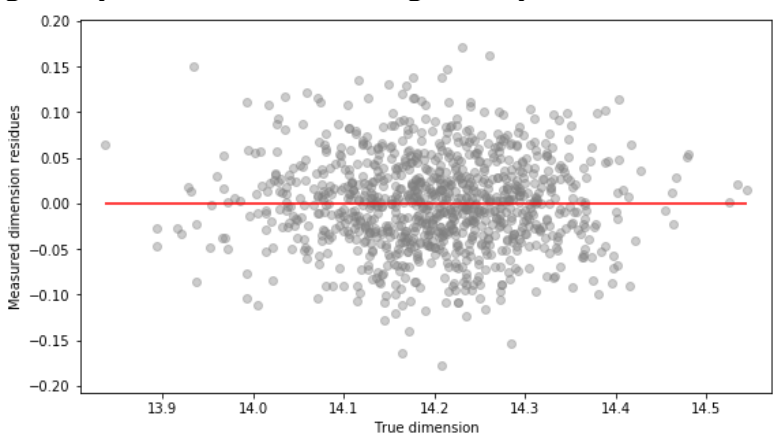

(a) measure. But no conclusion regarding the conformity of each part in the batch can be drawn. The confusion matrix is a relevant tool to synthesize all this information. It is also based on the idea that both the measured and the revised dimension are predictions of the truth. The two confusion matrices are shown in the Figure 4.

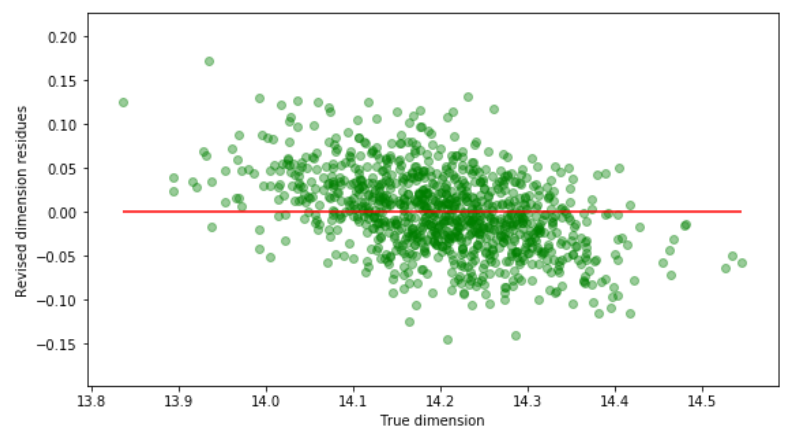

(b)

Figure 3: Measures and revised dimension residues.

(a) The measures residues with respect to the true dimension.

(b) The revised dimensions residues with respect to the true dimension.

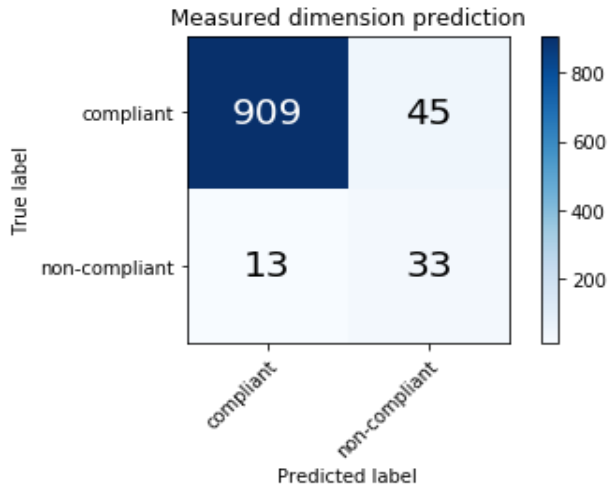

(a)

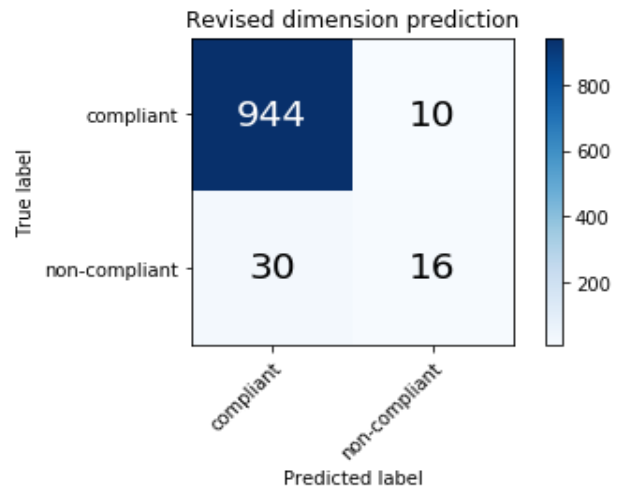

(b)

Figure 4: Confusion matrix of the different predictions.

The confusion matrix of the (a) measures and (b) revised dimensions.

In the top left corner is presented the number of right predictions about the actual conformity of the true dimension (True compliant); in the bottom right corner, the number of the right predictions about the actual non-conformity of the true dimension (True non-compliant). These right predictions constitute a diagonal in the matrix. The other diagonal groups all the errors: either when it is mistakenly believed the part is acceptable - in the bottom left corner (False compliant) - or when it is mistakenly believed the part is not acceptable - in the top right corner (False non-compliant).

The sum of True compliant and True non-compliant is higher using the revised dimensions and this is an encouraging result (960 against 942). However, these are many more False compliant (30 against 13) which leads to the conclusion that this method is "optimistic" as it tends to report more conformed value. To evaluate the true benefit with the confusion matrix, it is necessary for the industrialist to associate a cost for each of the four cases; or at least for the False compliant and False non-compliant which are respectively known as client risk and supplier risk.

The optimistic behavior identified can be explained by the linear relation between the revised dimension and the measure that has been given in equation (6). Indeed, applying the tolerance interval to the linearly transformed measure is equivalent to apply the linearly transformed tolerance interval to the measure. The new tolerance interval obtained after the Bayesian revision is called the acceptance interval. In our application its bounds are equal to 13.96 and 14.44 (against 14.0 and 14.2 originally). The width of the acceptance interval is increased; therefore the method reduces the number of rejects.

The Bayesian revision explains this enlargement as the measures are attracted by the a priori. In most of the common cases, it is in the tolerance interval and so, the small dimensions are increased and the large ones are reduced. The consequence of this optimistic attraction into the tolerance interval is an increase of the risk. Recall that the criterion to choose the revised dimension is to maximize the probability of the a posteriori distribution. However, this definition does not consider the probability to be out-of-tolerance which is a risk that the user should also be aware of. In the worst case shown in the Figure 5, the revised dimension falls on an extreme bound of the tolerance interval and, with Gaussian probability density function only, the probability to be out-ofit is 0.5 . To summarize, by considering the revised value or by 
applying the acceptance interval, industrialists are setting the admissible risk to mispredict the conformity up to 0.5 .

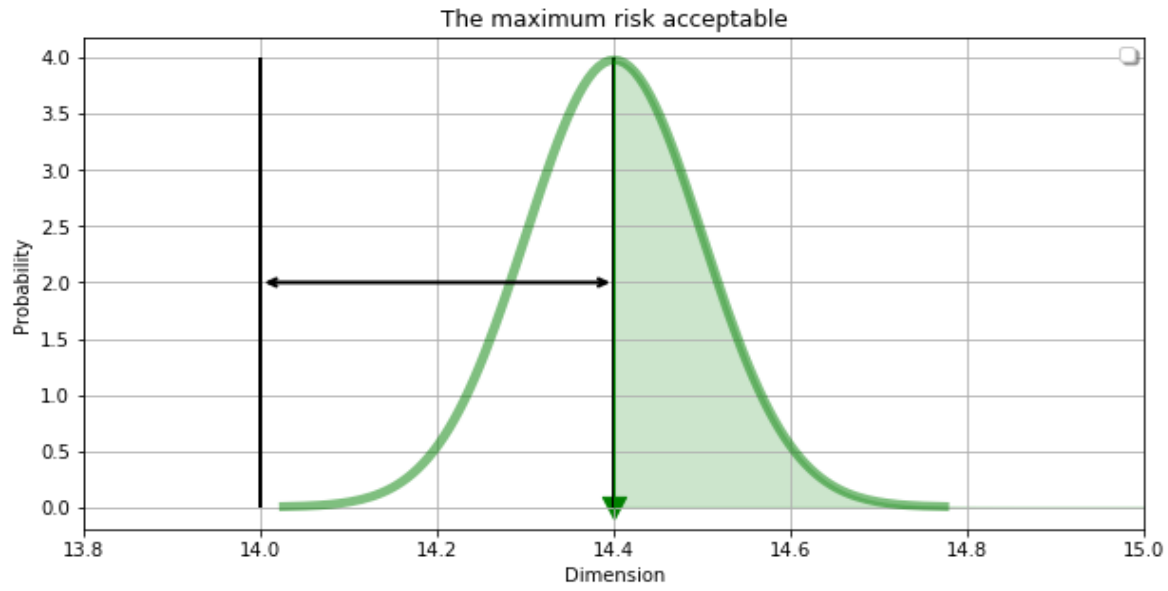

Figure 5: The maximum acceptable risk.

The large green curve is the a posteriori density. The green triangle is the revised dimension and the green area is the risk associated with this chosen value.

The Bayesian revision ends with an a posteriori probability density function which makes possible to compute the probability to be out-of-tolerance for the revised dimension. In this Gaussian modeling context, the acceptance interval has been determined analytically with the analytical expression of the mean. By proceeding experimentally, if the admissible risk is set to 0.5 then it is possible to identify all the revised value that are conformed without exceeding this probability: therefore, to identify the acceptance interval which must be applied on the measure to obtain the set of revised values which satisfy this conformity status. This approach may be used with any admissible risk threshold. Figure 6 shows an abacus to read the acceptance interval for any admissible risk threshold for the numerical values of this example.

Figure 6 is symmetric as the tolerance interval is centered on the true dimension. The basic results can be confirmed: if the wanted risk is null, then none of the measure meet the requirement, therefore none is compliant. If there is no limit to the acceptable risk then all of the measures are considered as compliant. The acceptance interval calculated before for a 0.5 risk with bounds equal to 13.96 and 14.44 can be found. An interesting result, read in reverse, is the revelation of the risk taking with the measurement process: in our application it is 0.18 .

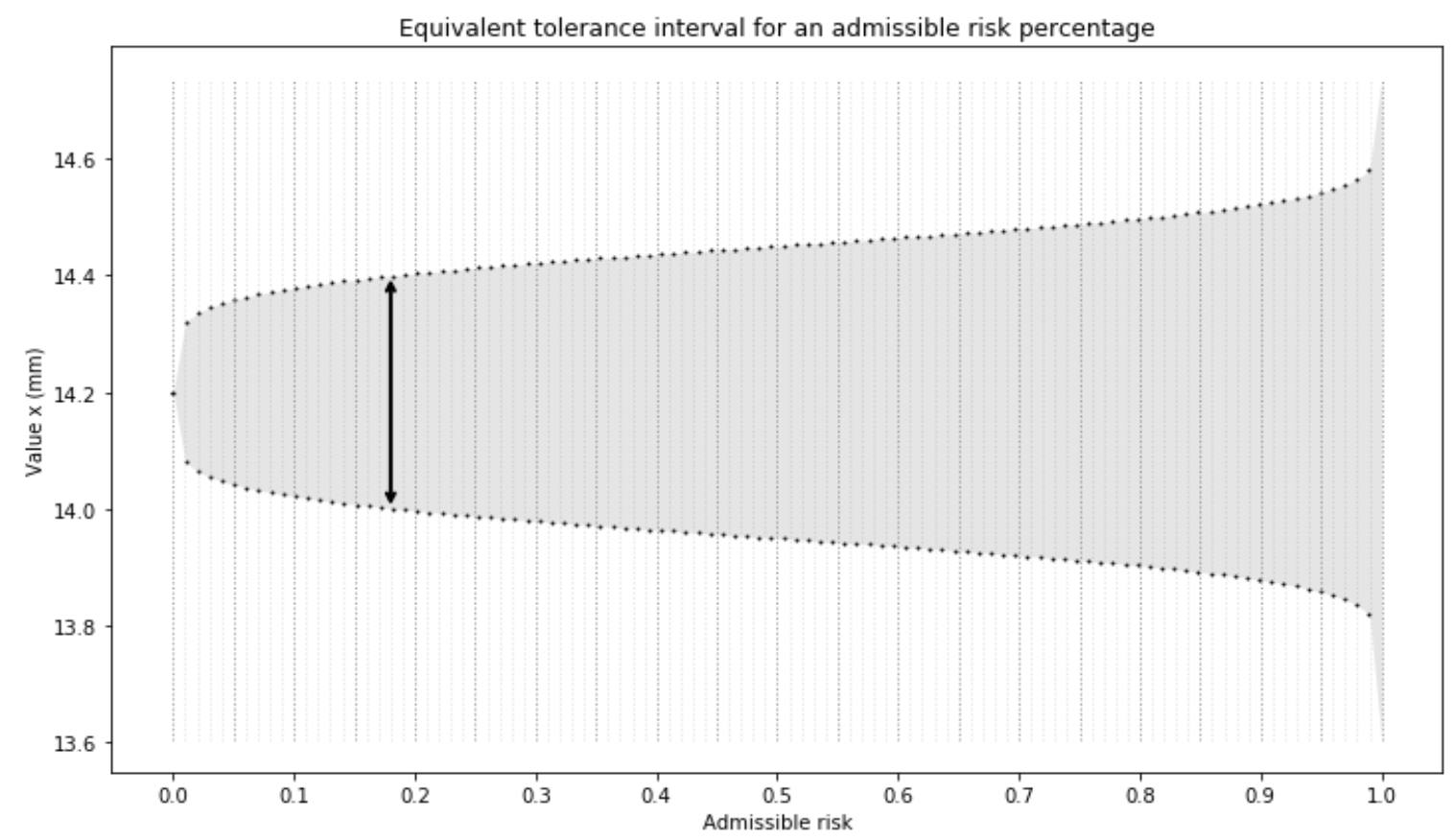

Figure 6: Acceptance interval for an admissible risk.

The black thick arrow reminds the original tolerance interval bounds at its equivalent risk. The black dots define the bounds of the acceptance interval included in the shaded area. 


\section{CONCLUSIONS}

In this paper, we present two methods that complement each other, which can be used by metrologists to consider the error random variable. The first deals with a batch of measures. It aims to correct the distribution which characterizes this batch from the error disturbances. The output is the new reference distribution to compute and estimate the usual quality metrics. The second method deals with measures one at a time. The output of the first method and the probability density function of the error are combined with the Bayesian statistics. It finally gives a new distribution to characterize the measure. The most probable dimension can be identified from this distribution, as well as the probability of being out-of-tolerance, for a given tolerance interval.

Both methods are applied to the same example; the data are generated using Monte Carlo simulation and it is observed that errors can be partially corrected. It also provides a diagram of the acceptance interval for a better control of the production risk. The concrete benefit is relative to the industrial application context, as it involves the actual costs of the client and the supplier risk. Remark that, if the Bayesian correction does not require the Gaussian model assumption, the deconvolution of non-parametric distribution is more challenging and will be studied in more detail in the future.

\section{ACKNOWLEDGMENTS}

The authors are grateful to J.-M. Pou and C. Dubois (DeltaMu) for their collaboration on this study and their innovative ideas. The operation « Proove of concept Services and Tools for Advanced Metrology » is cofinanced by the European Union in the context of the European Regional Development Fund (ERDF).

\section{REFERENCES}

[1] Les guides techniques du collège français de métrologie, Processus de mesure : évaluer les incertitudes 20 exemples. Afnor edition, 2018.

[2] Kane V, Process Capability Indices 1986, Journal of Quality Technology, 1986, pp. 41-52.

[3] Statistical methods in process management. Capability and performance, Part 1: general principles and concepts, NF ISO 22514-1 2014.

[4] M. L. Bailon, D. J. Hornrop, On the calculation of Convolutions with Gaussian Kernels CAMS Report 0506-46, 2006.

[5] A deconvolution algorithm for multi-echo functional MRI: Multi-echo Sparse: Paradigm Free Mapping. NeuroImage 202, 2019, 116081

[6] Sparse deconvolution via off-grid T.V minimization, Signal Processing, 170 (2020) 107406.

[7] Multichannel Semi-blind Deconvolution (MSBD) of seismic signals, Signal Processing 135 (2017), pp. 253-262

[8] Université de Reims Champagne Ardenne, MA 0804, 2013, Master 1, 2 Loi usuelles

[9] Dugué, D., « Calcul des probabilités », Encyclopædia Universalis, $1998, \S 4$.

[10] Boreux, J.-J., Parent, E., Bernier, J., Pratique du calcul bayesien. Springer, 2010.

[11] Bromiley, P. A., Products and Convolutions of Gaussian Probability Density Functions, 1.The Product of Two Univariate Gaussian PDFs, 2018, p.1 